\title{
Application of Vinyl Tris(trimethylsilyl)germanes in Pd-catalyzed Couplings
}

Zhizhong Wang and Stanislaw F. Wnuk

Department of Chemistry and Biochemistry, Florida International University, Miami, Florida

33199, USA

wnuk@fiu.edu

\section{SUPPORTING INFORMATION}

\section{CONTENTS}

1. Experimental procedures and characterization data for compounds:

- 2a and 2b (Germyldesulfonylation protocol, Procedure A): Page S2

- $\quad 4 a, 4 c, 4 d, 4 e$, and $4 \mathbf{f}$ (Hydrogermylation protocol, Procedure B): Pages S2-S4

- $\mathbf{5 a}(E)$, and $\mathbf{5 b}(E)$ (Coupling protocol under aqueous conditions, Method A): Pages S4-S6

- $\quad \mathbf{5 a}(E / Z), \mathbf{5} \mathbf{c}(E / Z), \mathbf{5 d}(E / Z), \mathbf{5 e}(E / Z), \mathbf{5 f}(E / Z), \mathbf{6 c}(E / Z)$, and $\mathbf{6} \mathbf{f}(E / Z)(C o u p l i n g$ protocol under anhydrous conditions, Method B): Pages S5-S8

2. ${ }^{1} \mathrm{H}$ NMR spectra for compounds:

- 5a(E): Page S10

- $\quad \mathbf{5 a}(E / Z)$ : Page S11

- $\quad \mathbf{5 c}(E / Z)$ : Page S12

- $\quad 5 d(E / Z)$ : Page S13

- $\quad 5 e(E / Z)$ : Page S14 


\section{General Procedures}

${ }^{1} \mathrm{H}\left(\mathrm{Me}_{4} \mathrm{Si}\right) \mathrm{NMR}$ spectra at $400 \mathrm{MHz},{ }^{13} \mathrm{C}\left(\mathrm{Me}_{4} \mathrm{Si}\right)$ at $100.6 \mathrm{MHz}$ and ${ }^{19} \mathrm{~F}\left(\mathrm{CCl}_{3} \mathrm{~F}\right)$ at 376.4 $\mathrm{MHz}$ were determined with solutions in $\mathrm{CDCl}_{3}$. Mass spectra (MS) were obtained by electron ionization (EI) technique. Reagent grade chemicals were used and solvents were dried by reflux over and distillation from $\mathrm{CaH}_{2}$ (except $\mathrm{THF} / \mathrm{Na} /$ benzophenone) under an argon atmosphere. TLC was performed on Merck kieselgel $60-\mathrm{F}_{254}$ and products were detected with $254 \mathrm{~nm}$ light or by development of color with $\mathrm{I}_{2}$. Merck kieselgel 60 (230-400 mesh) was used for column chromatography. Purity and identity of the products (crude and/or purified) were also established using a Hewlett-Packard (HP) GC/MS (EI) system with a HP 5973 mass selective detector [capillary column HP-5MS $(30 \mathrm{~m} \times 0.25 \mathrm{~mm} \times 25 \mu \mathrm{m})$ ]. Vinyl sulfones $\mathbf{1 a}$ and $\mathbf{1 b}$ were prepared as reported. ${ }^{4}$ Acetylenes $\mathbf{3 a}$ and $\mathbf{3 c - f}$ were commercially available.

(E)-2-Phenyl-1-[tris(trimethylsilyl)germyl]ethene (2a). Procedure A. Argon was bubbled through a solution of $\mathbf{1 a}(E)(450 \mathrm{mg}, 1.84 \mathrm{mmol})$ in anhydrous benzene or toluene $(25 \mathrm{~mL})$ for $15 \mathrm{~min} .\left(\mathrm{Me}_{3} \mathrm{Si}\right)_{3} \mathrm{GeH}(0.97 \mathrm{~mL}, 902 \mathrm{mg}, 3 \mathrm{mmol})$ and AIBN (308 $\mathrm{mg}, 1.84$ mmol) were added, and degassing of oxygen was continued for another $10 \mathrm{~min}$ and then the solution was heated at $80{ }^{\circ} \mathrm{C}$ (oil-bath) for $6 \mathrm{~h}$. The volatiles were evaporated, and the oily residue was column chromatographed (hexane) to give $\mathbf{2 a}^{4}$ (590 $\mathrm{mg}, 81 \%$ ): IR (neat) 2952, 1598, 1397, $1246 \mathrm{~cm}^{-1} ;{ }^{1} \mathrm{H}$ NMR $\delta 0.30(\mathrm{~s}, 27 \mathrm{H}), 6.72(\mathrm{~d}, J=18.5 \mathrm{~Hz}, 1 \mathrm{H}), 6.86(\mathrm{~d}, J=18.5$ $\mathrm{Hz}, 1 \mathrm{H}), 7.23$ (t, $J=7.1 \mathrm{~Hz}, 1 \mathrm{H}), 7.34(\mathrm{t}, J=7.5 \mathrm{~Hz}, 2 \mathrm{H}), 7.39$ (d, $J=7.0 \mathrm{~Hz}, 2 \mathrm{H}) ;{ }^{13} \mathrm{C} \mathrm{NMR}$ . $\delta 2.0,126.2,126.4,127.4(\mathrm{C} 1), 128.9,139.6,144.0(\mathrm{C} 2)$. GC-MS ( $\left.t_{\mathrm{R}} 19.99 \mathrm{~min}\right) \mathrm{m} / z 396(3.5$, $\left.\mathrm{M}^{+},{ }^{74} \mathrm{Ge}\right), 394\left(2.0, \mathrm{M}^{+},{ }^{72} \mathrm{Ge}\right), 392\left(1.5, \mathrm{M}^{+},{ }^{70} \mathrm{Ge}\right)$. Anal. Calcd for $\mathrm{C}_{17} \mathrm{H}_{34} \mathrm{GeSi}_{3}$ (395.32): $\mathrm{C}$, 51.65; H, 8.67. Found: C, 51.38; H, 8.89.

(E)-4-Phenyl-1-[tris(trimethylsilyl)germyl]-1-butene (2b). Treatment of $\mathbf{1 b}(E)(223 \mathrm{mg}$, $0.82 \mathrm{mmol})$ with $\left(\mathrm{Me}_{3} \mathrm{Si}\right)_{3} \mathrm{GeH}(0.265 \mathrm{~mL}, 246 \mathrm{mg}, 0.82 \mathrm{mmol})$ and AIBN (137 mg, 0.82 mmol) by procedure A gave $\mathbf{2 b}(177 \mathrm{mg}, 51 \%)$ as an oil: ${ }^{1} \mathrm{H}$ NMR $\delta 0.20$ (s, 27H), 2.44 ("q", $J=7.1 \mathrm{~Hz}, 2 \mathrm{H}), 2.71(\mathrm{t}, J=7.6 \mathrm{~Hz}, 2 \mathrm{H}), 5.73(\mathrm{~d}, J=17.8 \mathrm{~Hz}, 1 \mathrm{H}), 5.90(\mathrm{dt}, J=17.8,6.3 \mathrm{~Hz}$, 1H) 7.16-7.30 (m, 5H); ${ }^{13} \mathrm{C}$ NMR $\delta 1.8$ (TMS), 36.3 (C4), 39.4 (C3), $124.0(\mathrm{C} 1), 126.0$ (Ar), 128.6 (Ar), 128.9 (Ar), 142.5 (Ar), 145.7 (C2); MS m/z 424 (10, $\left.\mathrm{M}^{+}\right), 220$ (100). Anal. Calcd for $\mathrm{C}_{19} \mathrm{H}_{38} \mathrm{GeSi}_{3}$ (424.15): C, 53.90; H, 9.05. Found: C, 53.52; H, 9.29. 
(Z)-2-Phenyl-1-[tris(trimethylsilyl)germyl]ethene (4a). Procedure B. $\left(\mathrm{Me}_{3} \mathrm{Si}\right)_{3} \mathrm{GeH}$ $(0.323 \mathrm{~mL}, 300 \mathrm{mg}, 1 \mathrm{mmol})$ was added in one portion via a syringe to a stirred solution of 3a $(0.112 \mathrm{~mL}, 104 \mathrm{mg}, 1 \mathrm{mmol})$ in dry benzene $(3 \mathrm{~mL})$ at ambient temperature under nitrogen atmosphere. After $3 \mathrm{~h}$, the solvent was evaporated in vacuo and the oily residue was purified by flash chromatography on silica gel (hexane) to give $4 \mathbf{a}^{8 \mathrm{~b}}$ (388 $\left.\mathrm{mg}, 98 \%\right)$ : ${ }^{1} \mathrm{H}$ NMR $\delta 0.25(\mathrm{~s}, 27 \mathrm{H}), 6.12(\mathrm{~d}, J=13.6 \mathrm{~Hz}, 1 \mathrm{H}), 7.15-7.35(\mathrm{~m}, 5 \mathrm{H}), 7.41(\mathrm{~d}, J=13.6 \mathrm{~Hz}, 1 \mathrm{H})$.

(Z)-2-(4-Methoxyphenyl)-1-[tris(trimethylsilyl)germyl]ethene (4c). Treatment of 3c $(0.134 \mathrm{~mL}, 136 \mathrm{mg}, 1 \mathrm{mmol})$ with $\left(\mathrm{Me}_{3} \mathrm{Si}\right)_{3} \mathrm{GeH}(0.323 \mathrm{~mL}, 300 \mathrm{mg}, 1 \mathrm{mmol})$ and AIBN (16 $\mathrm{mg}, 0.10 \mathrm{mmol})$ by procedure $\mathrm{B}\left(3 \mathrm{~h} ; 82^{\circ} \mathrm{C}\right.$, oil bath) gave $4 \mathrm{c}(358 \mathrm{mg}, 84 \%)$ as a colorless oil: ${ }^{1} \mathrm{H}$ NMR $\delta 0.22$ (s, 27H), $3.84(\mathrm{~s}, 3 \mathrm{H}), 6.04(\mathrm{~d}, J=13.5 \mathrm{~Hz}, 1 \mathrm{H}), 6.88(\mathrm{~d}, J=8.6 \mathrm{~Hz}, 2 \mathrm{H})$, $7.35(\mathrm{~d}, J=8.6 \mathrm{~Hz}, 2 \mathrm{H}), 7.39$ (d, $J=13.5 \mathrm{~Hz}, 1 \mathrm{H}) ;{ }^{13} \mathrm{C} \mathrm{NMR} \delta 2.2,55.7,114.0,125.2,129.5$, 133.9, 143.6, 159.2; MS m/z $426\left(15, \mathrm{M}^{+}\right), 220$ (100). Anal. Calcd for $\mathrm{C}_{18} \mathrm{H}_{36} \mathrm{GeOSi}_{3}$ (426.13): C, 50.83; H, 8.53. Found: C, 50.49; H, 8.61.

(Z)-2-(2-Trifluoromethylphenyl)-1-[tris(trimethylsilyl)germyl]ethene (4d). Treatment of $3 \mathbf{d}(0.144 \mathrm{~mL}, 175 \mathrm{mg}, 1 \mathrm{mmol})$ with $\left(\mathrm{Me}_{3} \mathrm{Si}\right)_{3} \mathrm{GeH}(0.323 \mathrm{~mL}, 300 \mathrm{mg}, 1 \mathrm{mmol})$ and AIBN (16 mg, $0.10 \mathrm{mmol})$ by procedure B $\left(3 \mathrm{~h} ; 82^{\circ} \mathrm{C}\right.$, oil bath) gave $4 \mathbf{d}(371 \mathrm{mg}, 80 \%)$ as a colorless oil: ${ }^{1} \mathrm{H}$ NMR $\delta 0.16(\mathrm{~s}, 27 \mathrm{H}), 6.30(\mathrm{~d}, J=13.7 \mathrm{~Hz}, 1 \mathrm{H}), 7.34-7.67(\mathrm{~m}, 5 \mathrm{H}) ;{ }^{13} \mathrm{C}$ NMR $\delta 2.0,124.7(\mathrm{q}, J=274.0 \mathrm{~Hz}), 126.1(\mathrm{q}, J=5.6 \mathrm{~Hz}), 127.3,128.5(\mathrm{q}, J=29.6 \mathrm{~Hz})$, 130.4, 131.3, 132.3, 140.6, 140.8; ${ }^{19} \mathrm{~F}$ NMR $\delta$-60.83 (s); MS m/z 464 (20, M $), 220$ (100). Anal. Calcd for $\mathrm{C}_{18} \mathrm{H}_{33} \mathrm{~F}_{3} \mathrm{GeSi}_{3}$ (464.11): C, 46.66; H, 7.18. Found: C, 47.04; H, 7.44.

(Z)-2-(4-Trifluoromethylphenyl)-1-[tris(trimethylsilyl)germyl]ethene (4e). Treatment of $3 \mathbf{e}(0.173 \mathrm{~mL}, 175 \mathrm{mg}, 1 \mathrm{mmol})$ with $(\mathrm{TMS})_{3} \mathrm{GeH}(0.323 \mathrm{~mL}, 300 \mathrm{mg}, 1 \mathrm{mmol})$ and AIBN (16 mg, $0.10 \mathrm{mmol})$ by procedure $\mathrm{B}\left(3 \mathrm{~h} ; 82^{\circ} \mathrm{C}\right.$, oil bath ) gave $4 \mathrm{e}(394 \mathrm{mg}, 85 \%)$ as a colorless oil: ${ }^{1} \mathrm{H}$ NMR $\delta 0.16(\mathrm{~s}, 27 \mathrm{H}), 6.32(\mathrm{~d}, J=13.7 \mathrm{~Hz}, 1 \mathrm{H}), 7.44(\mathrm{~d}, J=13.7 \mathrm{~Hz}, 1 \mathrm{H})$, $7.48(\mathrm{~d}, J=8.1 \mathrm{~Hz}, 2 \mathrm{H}), 7.58(\mathrm{~d}, J=8.1 \mathrm{~Hz}, 2 \mathrm{H}) ;{ }^{13} \mathrm{C} \mathrm{NMR} \delta 2.2,124.7(\mathrm{q}, J=271.9 \mathrm{~Hz})$, $125.5(\mathrm{q}, J=3.7 \mathrm{~Hz}), 128.5,129.3(\mathrm{q}, J=32.4 \mathrm{~Hz}), 131.5,142.8,144.6 ;{ }^{19} \mathrm{~F}$ NMR $\delta-62.84$ (s); MS m/z 464 (2, $\mathrm{M}^{+}$), 220 (100). Anal. Calcd for $\mathrm{C}_{18} \mathrm{H}_{33} \mathrm{~F}_{3} \mathrm{GeSi}_{3}$ (464.11): C, 46.66; H, 7.18. Found: C, 46.31; H, 7.27. 
(Z)-1-[Tris(trimethylsilyl)germyl]octene. (4f). Treatment of $\mathbf{3 f}(0.152 \mathrm{~mL}, 114 \mathrm{mg}, 1$ mmol) with $\left(\mathrm{Me}_{3} \mathrm{Si}\right)_{3} \mathrm{GeH}(0.323 \mathrm{~mL}, 300 \mathrm{mg}, 1 \mathrm{mmol})$ and AIBN (16 mg, $\left.0.10 \mathrm{mmol}\right)$ by procedure B $\left(3 \mathrm{~h}, 82{ }^{\circ} \mathrm{C}\right.$, oil bath ) gave $4 \mathrm{f}^{8 \mathrm{~b}}(384 \mathrm{mg}, 95 \%):{ }^{1} \mathrm{H}$ NMR: $\delta 0.21(\mathrm{~s}, 27 \mathrm{H})$, 0.88-0.91 (m, 3H), 1.25-1.43 (m, 8H), 2.05 ("q", $J=7.4$ Hz, 2H), 5.67 (dt, $J=12.0,1.4 \mathrm{~Hz}$, $1 \mathrm{H}), 6.33(\mathrm{dt}, J=12.0,6.8 \mathrm{~Hz}, 1 \mathrm{H})$.

(E)-1,2-Diphenylethene (5a). Method A. A solution of $\mathrm{NaOH}(12 \mathrm{mg}, 0.3 \mathrm{mmol})$ and $\mathrm{H}_{2} \mathrm{O}_{2}(30 \%$ solution, $20 \mu \mathrm{L}, 0.20 \mathrm{mmol})$ in deionized $\mathrm{H}_{2} \mathrm{O}(0.4 \mathrm{~mL})$ was added to a stirred solution of $2 \mathbf{a}(25 \mathrm{mg}, 0.063 \mathrm{mmol})$ in THF ( $3 \mathrm{~mL})$ at ambient temperature. After 15 minutes, iodobenzene $(11 \mu \mathrm{L}, 20 \mathrm{mg}, 0.095 \mathrm{mmol})$ and $\mathrm{Pd}\left(\mathrm{PPh}_{3}\right)_{4}(7 \mathrm{mg}, 0.006 \mathrm{mmol})$ were added and the resulting brownish mixture was heated at $45^{\circ} \mathrm{C}$ (oil bath) for $10 \mathrm{~h}$. The volatiles were evaporated and the residue was partitioned $\left(\mathrm{H}_{2} \mathrm{O} / \mathrm{CHCl}_{3}\right)$. The organic layer was dried $\left(\mathrm{MgSO}_{4}\right)$, evaporated and purified by column chromatography (hexane) to give $\mathbf{5 a}(5.7 \mathrm{mg}$, $50 \%$ ) with data identical to a commercial sample.

Treatment of $2 \mathbf{a}(25 \mathrm{mg}, 0.063 \mathrm{mmol})$ with bromobenzene $(11 \mu \mathrm{L}, 16 \mathrm{mg}, 0.10 \mathrm{mmol})$ by Method A gave 5a (10 mg, 88\%).

Treatment of $2 \mathrm{a}(25 \mathrm{mg}, 0.063 \mathrm{mmol})$ with chlorobenzene $(10 \mu \mathrm{L}, 11 \mathrm{mg}, 0.10 \mathrm{mmol})$ by Method A gave 5a (4 mg, 37\%): GC-MS ( $\left.t_{\mathrm{R}} 16.6 \mathrm{~min}\right) \mathrm{m} / \mathrm{z} 180\left(100, \mathrm{M}^{+}\right)$. The (E,E)-1,4-diphenyl-1,3-butadiene (3 $\mathrm{mg}, 23 \%)$ was also isolated with data identical to a commercial sample and as reported: ${ }^{16} \mathrm{GC}-\mathrm{MS}\left(t_{\mathrm{R}} 20.2 \mathrm{~min}\right) \mathrm{m} / z 206\left(100, \mathrm{M}^{+}\right)$.

Treatment of $2 \mathbf{a}(25 \mathrm{mg}, 0.063 \mathrm{mmol})$ with phenyl trifluoromethanesulfonate $(16 \mu \mathrm{L}$, $22 \mathrm{mg}, 0.095 \mathrm{mmol})$ by Method A gave 5a (1.7 $\mathrm{mg}, 15 \%)$. The $(E, E)-1,4-$ diphenyl-1,3-butadiene ${ }^{16}(4.4 \mathrm{mg}, 34 \%)$ was also isolated.

Method B. KH $(12 \mathrm{mg}, 0.3 \mathrm{mmol})$ and $t$ - $\mathrm{BuOOH}(5.0-6.0 \mathrm{M}$ solution, $37.8 \mu \mathrm{L}, 0.19$ mmol) were added to a stirred solution of $\mathbf{2 a}(25 \mathrm{mg}, 0.063 \mathrm{mmol})$ in THF (3 mL) at ambient temperature. After $15 \mathrm{~min}$, iodobenzene $(11 \mu \mathrm{L}, 20 \mathrm{mg}, 0.095 \mathrm{mmol})$ and $\mathrm{Pd}\left(\mathrm{PPh}_{3}\right)_{4}(7 \mathrm{mg}$, $0.006 \mathrm{mmol}$ ) were added and the resulting brownish mixture was heated at $45^{\circ} \mathrm{C}$ (oil bath) for $10 \mathrm{~h}$. The volatiles were evaporated and the residue was partitioned $\left(\mathrm{H}_{2} \mathrm{O} / \mathrm{CHCl}_{3}\right)$. The organic layer was dried $\left(\mathrm{MgSO}_{4}\right)$, evaporated and purified by column chromatography (hexane) to give $\mathbf{5 a}(9.1 \mathrm{mg}, 80 \%)$ with data identical to a commercial sample.

Treatment of $2 \mathrm{a}(25 \mathrm{mg}, 0.063 \mathrm{mmol})$ with bromobenzene $(11 \mu \mathrm{L}, 16 \mathrm{mg}, 0.10 \mathrm{mmol})$ 
by Method B gave 5a (5.7 mg, 50\%).

Treatment of $2 \mathrm{a}(25 \mathrm{mg}, 0.063 \mathrm{mmol})$ with chlorobenzene $(10 \mu \mathrm{L}, 11 \mathrm{mg}, 0.10 \mathrm{mmol})$ by Method B gave 5a (1.1 mg, 10\%). The (E,Z)-1,4-diphenyl-1,3-butadiene ${ }^{17}[0.5 \mathrm{mg}, 4 \%)$ : GC-MS $\left.\left(t_{\mathrm{R}} 18.7 \mathrm{~min}\right) \mathrm{m} / z 206\left(100, \mathrm{M}^{+}\right)\right]$and the $(E, E)$-1,4-diphenyl-1,3-butadiene ${ }^{16}(3.3 \mathrm{mg}$, $25 \%)$ were also isolated.

Treatment of 2a (25 mg, $0.063 \mathrm{mmol})$ with phenyl trifluoromethanesulfonate (16 $\mu \mathrm{L}, 22$ $\mathrm{mg}, 0.095 \mathrm{mmol})$ by Method B gave 5a $(2.3 \mathrm{mg}, 20 \%)$. The $(E, Z)-(1.6 \mathrm{mg}, 12 \%)$ and (E,E)-1,4-diphenyl-1,3-butadiene (3.0 mg, 24\%) were also isolated.

$(\boldsymbol{E}, \boldsymbol{Z})$-1,2-Diphenylethene (5a). Treatment of $\mathbf{4 a}(45 \mathrm{mg}, 0.11 \mathrm{mmol})$ with iodobenzene $(19 \mu \mathrm{L}, 35 \mathrm{mg}, 0.17 \mathrm{mmol})$ by Method A gave 5a $(E / Z, 33 / 67 ; 12 \mathrm{mg}, 60 \%)$ with data identical to commercial sample. GC-MS ( $\left.t_{\mathrm{R}} 13.9 \mathrm{~min}, \mathrm{Z} ; t_{\mathrm{R}} 16.6 \mathrm{~min}, E\right) \mathrm{m} / \mathrm{z} 180$ $\left(100, \mathrm{M}^{+}\right)$.

Treatment of $4 \mathbf{a}(25 \mathrm{mg}, 0.063 \mathrm{mmol})$ with bromobenzene $(11 \mu \mathrm{L}, 16 \mathrm{mg}, 0.095 \mathrm{mmol})$ by Method A gave 5a $(E / Z, 1: 1 ; 6 \mathrm{mg}, 53 \%)$. Diphenyl $(0.6 \mathrm{mg}, 4 \%$; e.g. $8 \%$ consumption of iodobenzene) was also isolated: GC-MS ( $\left.t_{\mathrm{R}} 11.3 \mathrm{~min}\right), \mathrm{m} / z 154\left(100, \mathrm{M}^{+}\right)$.

Treatment of $4 \mathbf{a}(25 \mathrm{mg}, 0.063 \mathrm{mmol})$ with chlorobenzene $(10 \mu \mathrm{L}, 11 \mathrm{mg}, 0.095 \mathrm{mmol})$ by Method A gave 5a $(E / Z, 2: 1 ; 5 \mathrm{mg}, 44 \%)$. The isomers of 1,4-diphenyl-1,3-butadiene (1.0 $\mathrm{mg}, 8 \%$ ) were also isolated.

Treatment of $4 \mathbf{a}(25 \mathrm{mg}, 0.063 \mathrm{mmol})$ with phenyl trifluoromethanesulfonate $(16 \mu \mathrm{L}, 22$ mg, $0.095 \mathrm{mmol}$ ) by Method A gave 5a (3.8 mg, 33\%; $E / Z, 99: 1)$. The $E / E$ and $E / Z$ isomers of 1,4-diphenyl-1,3-butadiene (4.0 mg, 31\%) were also isolated.

Treatment of 4a $(22.6 \mathrm{mg}, 0.057 \mathrm{mmol})$ with iodobenzene $(9.9 \mu \mathrm{L}, 18 \mathrm{mg}, 0.09 \mathrm{mmol})$ by Method B gave 5a $(E / Z, 3 / 97 ; 8.5 \mathrm{mg}, 83 \%)$.

Treatment of $4 \mathbf{a}(22.6 \mathrm{mg}, 0.057 \mathrm{mmol})$ with bromobenzene $(9.9 \mu \mathrm{L}, 14.4 \mathrm{mg}, 0.09$ mmol) by Method B gave 5a $(E / Z, 8 / 92 ; 8 \mathrm{mg}, 78 \%)$.

(E)-1,4-Diphenyl-1-butene (5b). Treatment of $\mathbf{2 b}(30 \mathrm{mg}, 0.071 \mathrm{mmol})$ with iodobenzene $(12 \mu \mathrm{L}, 22.5 \mathrm{mg}, 0.11 \mathrm{mmol})$ by Method A gave $\mathbf{5 b}(7.5 \mathrm{mg}, 51 \%)$ with data as reported. ${ }^{18}{ }^{1} \mathrm{H}$ NMR $\delta 2.53$ ("q", $\left.J=7.8 \mathrm{~Hz}, 2 \mathrm{H}\right), 2.79$ (t, $\left.J=7.8 \mathrm{~Hz}, 2 \mathrm{H}\right), 6.26$ (dt, $J=15.8$, $6.7 \mathrm{~Hz}, 1 \mathrm{H}), 6.42$ (d, $J=15.8 \mathrm{~Hz}, 1 \mathrm{H}), 7.18-7.34(\mathrm{~m}, 10 \mathrm{H}) ;{ }^{13} \mathrm{C} \mathrm{NMR} \delta 34.9,35.8,125.9$, 126.0, 126.9, 128.3, $128.5(2 \times), 129.9,130.3,137.7,141.7$; GC-MS ( $\left.t_{\mathrm{R}} 16.3 \mathrm{~min}\right), \mathrm{m} / z 208$ 
$\left(100, \mathrm{M}^{+}\right)$. Anal. Calcd for $\mathrm{C}_{16} \mathrm{H}_{16}$ (208.30): C, 92.26; H, 7.74. Found: 91.89; H, 7.39.

Diphenyl $(0.5 \mathrm{mg}, 3 \%$; e.g. $6 \%$ consumption of iodobenzene) was also isolated: GC-MS ( $\left.t_{\mathrm{R}} 11.3 \mathrm{~min}\right), m / z 154\left(100, \mathrm{M}^{+}\right)$.

Treatment of $\mathbf{2 b}(14 \mathrm{mg}, 0.033 \mathrm{mmol})$ with bromobenzene $(5 \mu \mathrm{L}, 8 \mathrm{mg}, 0.05 \mathrm{mmol})$ by Method A gave 5b (3.4 mg, 73\%).

Treatment of $\mathbf{2 b}(18 \mathrm{mg}, 0.042 \mathrm{mmol})$ with iodobenzene $(7.4 \mu \mathrm{L}, 13 \mathrm{mg}, 0.064 \mathrm{mmol})$ by Method B gave $\mathbf{5 b}(7 \mathrm{mg}, 80 \%)$.

(E,Z)-1-(4-Methoxyphenyl)-2-phenylethene (5c). Treatment of 4c (53.6 mg, 0.126 mmol) with iodobenzene $(22 \mu \mathrm{L}, 40 \mathrm{mg}, 0.19 \mathrm{mmol})$ by Method A gave 5c $(E / Z, 13: 87 ; 24$ mg, 90\%) with data as reported. ${ }^{16} \mathbf{5 c}(E):{ }^{1} \mathrm{H}$ NMR $\delta 3.84(\mathrm{~s}, 3 \mathrm{H}), 6.91(\mathrm{~d}, J=8.8 \mathrm{~Hz}, 2 \mathrm{H})$, $6.98(\mathrm{~d}, J=16.4 \mathrm{~Hz}, 1 \mathrm{H}), 7.07(\mathrm{~d}, J=16.4 \mathrm{~Hz}, 1 \mathrm{H}), 7.23-7.50(\mathrm{~m}, 7 \mathrm{H}) ;{ }^{13} \mathrm{C} \mathrm{NMR} \delta 55.3$, 114.1, 126.2, 126.6, 127.2, 127.7, 128.2, 128.6, 130.1, 137.6, 159.2; MS m/z $210\left(100, \mathrm{M}^{+}\right)$. 5c(Z): ${ }^{1} \mathrm{H}$ NMR $\delta 3.81(\mathrm{~s}, 3 \mathrm{H}), 6.53(\mathrm{~d}, J=12.4 \mathrm{~Hz}, 1 \mathrm{H}), 6.57$ (d, $\left.J=12.4 \mathrm{~Hz}, 1 \mathrm{H}\right), 6.78(\mathrm{~d}, J$ $=8.7 \mathrm{~Hz}, 2 \mathrm{H}), 7.20-7.31(\mathrm{~m}, 7 \mathrm{H}) ;{ }^{13} \mathrm{C} \mathrm{NMR} \delta 55.6,114.0,127.3,128.6,129.2,129.2,130.1$, 130.2, 130.6, 138.0, 159.1; MS $m / z 210\left(100, \mathrm{M}^{+}\right)$.

Diphenyl (1.5 mg, 5\%; e.g. 10\% consumption of iodobenzene) was also isolated: GC-MS $\left[t_{\mathrm{R}} 11.3 \mathrm{~min}, \mathrm{~m} / z 154\left(100, \mathrm{M}^{+}\right) ; t_{\mathrm{R}} 17.4 \mathrm{~min}\right.$ and $t_{\mathrm{R}} 19.9 \min$ for $\mathbf{5 c}(\mathrm{Z})$ and $\mathbf{5 c}(E)$, respectively, $\left.m / z 210\left(100, \mathrm{M}^{+}\right)\right]$.

Treatment of $4 \mathbf{c}(26.8 \mathrm{mg}, 0.063 \mathrm{mmol})$ with bromobenzene $(11 \mu \mathrm{L}, 16 \mathrm{mg}, 0.095$ mmol) by Method A gave 5c (E/Z, 64:36; $6.6 \mathrm{mg}, 50 \%)$.

Treatment of $4 \mathbf{c}(53.6 \mathrm{mg}, 0.126 \mathrm{mmol})$ with iodobenzene $(22 \mu \mathrm{L}, 40 \mathrm{mg}, 0.19 \mathrm{mmol})$ by Method B gave 5c $(E / Z, 3: 97 ; 25.4 \mathrm{mg}, 96 \%)$.

Treatment of $4 \mathbf{c}(26.8 \mathrm{mg}, 0.063 \mathrm{mmol})$ with bromobenzene $(11 \mu \mathrm{L}, 16 \mathrm{mg}, 0.095$ mmol) by Method B gave 5c $(E / Z, 2: 98 ; 1.3 \mathrm{mg}, 10 \%)$. The mixture of isomers of 1,4-di-(4-methoxyphenyl)-1,3-butadiene ${ }^{19}$ (5 mg, 30\%): GC-MS ( $t_{\mathrm{R}} 24.9 \mathrm{~min}, t_{\mathrm{R}} 25.1 \mathrm{~min}$ and $\left.t_{\mathrm{R}} 26.5 \mathrm{~min}\right) \mathrm{m} / \mathrm{z} 266\left(100, \mathrm{M}^{+}\right)$were also isolated.

(E,Z)-1-(2-Trifluoromethylphenyl)-2-phenylethene (5d). Treatment of 4d (29.2 mg, $0.063 \mathrm{mmol})$ with iodobenzene $(11 \mu \mathrm{L}, 20 \mathrm{mg}, 0.095 \mathrm{mmol})$ by Method A gave $\mathbf{5 d}^{20}(E / Z$, 21:79; $6.4 \mathrm{mg}, 41 \%) .5 \mathrm{~d}(E):{ }^{1} \mathrm{H}$ NMR $\delta$ 7.07-7.82 (m, $\left.11 \mathrm{H}\right) ;{ }^{19} \mathrm{~F}$ NMR $\delta$-59.79 (s). 5d(Z): ${ }^{1} \mathrm{H}$ NMR $\delta 6.75(\mathrm{~d}, J=12.2 \mathrm{~Hz}, 1 \mathrm{H}), 6.85(\mathrm{~d}, J=12.2 \mathrm{~Hz}, 1 \mathrm{H}), 7.07-7.73(\mathrm{~m}, 7 \mathrm{H}) ;{ }^{13} \mathrm{C} \mathrm{NMR}$ 
$\delta 124.7(\mathrm{q}, J=273.7 \mathrm{~Hz}), 126.3(\mathrm{q}, J=5.3 \mathrm{~Hz}), 127.3,127.5,127.7,128.5,128.8(\mathrm{q}, J=29.0$ $\mathrm{Hz}), 129.6,131.7,131.9,132.6,136.6,137.1$ (d, $J=2.3 \mathrm{~Hz}) ;{ }^{19} \mathrm{~F}$ NMR $\delta$-61.56 (s); MS m/z $248\left(100, \mathrm{M}^{+}\right)$.

Treatment of 4d $(29.2 \mathrm{mg}, 0.063 \mathrm{mmol})$ with bromobenzene $(11 \mu \mathrm{L}, 16 \mathrm{mg}, 0.095$ mmol) by Method A gave 5d (E/Z, 76:24; $4.7 \mathrm{mg}, 30 \%)$.

Treatment of 4d $(29.2 \mathrm{mg}, 0.063 \mathrm{mmol})$ with iodobenzene $(11 \mu \mathrm{L}, 20 \mathrm{mg}, 0.095 \mathrm{mmol})$ by Method B gave 5d (E/Z, 1:99; $12 \mathrm{mg}, 77 \%)$.

Treatment of $4 \mathbf{d}(29.2 \mathrm{mg}, 0.063 \mathrm{mmol})$ with bromobenzene $(11 \mu \mathrm{L}, 16 \mathrm{mg}, 0.095$ mmol) by Method B gave 5d (E/Z, 3:97; $2.3 \mathrm{mg}, 15 \%)$. The by-products tentatively assigned as isomers of 1,4-di-(2-trifluoromethylphenyl)-1,3-butadiene (4.3 $\mathrm{mg}, 20 \%)$ were also isolated: GC-MS ( $t_{\mathrm{R}} 17.2 \mathrm{~min}, t_{\mathrm{R}} 17.5 \mathrm{~min}$ and $\left.t_{\mathrm{R}} 19.1 \mathrm{~min}\right) \mathrm{m} / z 342\left(100, \mathrm{M}^{+}\right)$.

(E,Z)-1-(4-Trifluoromethylphenyl)-2-phenylethene (5e). Treatment of 4e (29.2 $\mathrm{mg}$, $0.063 \mathrm{mmol})$ with iodobenzene $(11 \mu \mathrm{L}, 20 \mathrm{mg}, 0.095 \mathrm{mmol})$ by Method A gave 5e $(E / Z$, 55:45; $12.5 \mathrm{mg}, 80 \%) .5 \mathrm{e}(E):{ }^{21 \mathrm{a}}{ }^{1} \mathrm{H}$ NMR $\delta 7.14(\mathrm{~d}, J=16.3 \mathrm{~Hz}, 1 \mathrm{H}), 7.22(\mathrm{~d}, J=16.3 \mathrm{~Hz}$, 1H), 7.31-7.63 (m, 9H); ${ }^{19} \mathrm{~F}$ NMR $\delta$-62.90 (s); MS m/z $248\left(100, \mathrm{M}^{+}\right) .5 \mathbf{e}(Z):{ }^{21 \mathrm{~b}}{ }^{1} \mathrm{H}$ NMR $\delta$ $6.62(\mathrm{~d}, J=12.3 \mathrm{~Hz}, 1 \mathrm{H}), 6.75(\mathrm{~d}, J=12.3 \mathrm{~Hz}, 1 \mathrm{H}), 7.23-7.50(\mathrm{~m}, 9 \mathrm{H}) ;{ }^{19} \mathrm{~F}$ NMR $\delta-62.98(\mathrm{~s})$; $\operatorname{MS~} m / z 248\left(100, \mathrm{M}^{+}\right)$.

Treatment of $4 \mathbf{e}(29.2 \mathrm{mg}, 0.063 \mathrm{mmol})$ with bromobenzene $(11 \mu \mathrm{L}, 16 \mathrm{mg}, 0.095 \mathrm{mmol})$ by Method A gave 5e (E/Z, 99:1; $10.6 \mathrm{mg}, 68 \%)$.

Treatment of $4 \mathbf{e}(29.2 \mathrm{mg}, 0.063 \mathrm{mmol})$ with chlorobenzene $(10 \mu \mathrm{L}, 11 \mathrm{mg}, 0.095 \mathrm{mmol})$ by Method A gave 5e $(E / Z, 56: 44 ; 2.3 \mathrm{mg}, 15 \%)$. The isomers of 1,4-di-(4-trifluoromethyl phenyl)-1,3-butadiene ${ }^{22}$ (6.3 mg, 29\%): GC-MS ( $t_{\mathrm{R}} 13.5 \mathrm{~min}, t_{\mathrm{R}} 18.3 \mathrm{~min}$ and $\left.t_{\mathrm{R}} 18.4 \mathrm{~min}\right)$ $m / z 342\left(100, \mathrm{M}^{+}\right)$were also isolated.

Treatment of $4 \mathbf{e}(29.2 \mathrm{mg}, 0.063 \mathrm{mmol})$ with iodobenzene $(11 \mu \mathrm{L}, 20 \mathrm{mg}, 0.095 \mathrm{mmol})$ by Method B gave 5e $(E / Z, 5: 95 ; 11 \mathrm{mg}, 71 \%)$.

Treatment of $4 \mathrm{e}(29.2 \mathrm{mg}, 0.063 \mathrm{mmol})$ with bromobenzene $(11 \mu \mathrm{L}, 16 \mathrm{mg}, 0.095 \mathrm{mmol})$ by Method B gave 5e (E/Z, 1:99; 9 mg, 58\%).

$(\boldsymbol{E}, \boldsymbol{Z})$-1-Phenyl-1-octene (5f). Treatment of $\mathbf{4 f}(25.5 \mathrm{mg}, 0.063 \mathrm{mmol})$ with iodobenzene $(11 \mu \mathrm{L}, 20 \mathrm{mg}, 0.095 \mathrm{mmol})$ by Method A gave $\mathbf{5 f}(E / Z, 45: 55 ; 4.3 \mathrm{mg}, 36 \%)$ with data as reported. 5f(E): ${ }^{16}{ }^{1} \mathrm{H}$ NMR $\delta 0.91(\mathrm{t}, J=6.0 \mathrm{~Hz}, 3 \mathrm{H}), 1.32-1.53(\mathrm{~m}, 8 \mathrm{H}), 2.22(\mathrm{q}, J=6.0 \mathrm{~Hz}$, 
2H), $6.24(\mathrm{dt}, J=15.0,6.0 \mathrm{~Hz}, 1 \mathrm{H}), 6.39(\mathrm{~d}, J=15.0 \mathrm{~Hz}, 1 \mathrm{H}), 7.17-7.37(\mathrm{~m}, 5 \mathrm{H}) ;{ }^{13} \mathrm{C} \mathrm{NMR} \delta$ 14.2, 22.7, 29.0, 29.4, 31.9, 33.2, 126.0, 126.8, 128.5, 129.7, 131.4, 138.1. 5f(Z): ${ }^{.231} \mathrm{H}$ NMR $\delta$ $0.91(\mathrm{t}, J=6.0 \mathrm{~Hz}, 3 \mathrm{H}), 1.32-1.53(\mathrm{~m}, 8 \mathrm{H}), 2.35(\mathrm{q}, J=7.4 \mathrm{~Hz}, 2 \mathrm{H}), 5.69$ (dt, $J=11.7,7.2$ $\mathrm{Hz}, 1 \mathrm{H}), 6.42(\mathrm{~d}, J=11.7 \mathrm{~Hz}, 1 \mathrm{H}), 7.18-7.35(\mathrm{~m}, 5 \mathrm{H})$; MS m/z $188\left(100, \mathrm{M}^{+}\right)$. Anal. Calcd for $\mathrm{C}_{14} \mathrm{H}_{20}$ (188.31): C, 89.29; H, 10.71. Found: C, 88.93; H, 10.42.

Treatment of $\mathbf{4 f}(25.5 \mathrm{mg}, 0.063 \mathrm{mmol})$ with bromobenzene $(11 \mu \mathrm{L}, 16 \mathrm{mg}, 0.095$ mmol) by Method A gave $\mathbf{5 f}(E / Z, 33: 67 ; 7.6 \mathrm{mg}, 64 \%)$.

Treatment of $\mathbf{4 f}(25.5 \mathrm{mg}, 0.063 \mathrm{mmol})$ with iodobenzene $(11 \mu \mathrm{L}, 20 \mathrm{mg}, 0.095 \mathrm{mmol})$ by Method B gave $\mathbf{5 f}(E / Z, 25: 75 ; 7.0 \mathrm{mg}, 60 \%)$.

Treatment of $4 \mathbf{f}(25.5 \mathrm{mg}, 0.063 \mathrm{mmol})$ with bromobenzene $(11 \mu \mathrm{L}, 16 \mathrm{mg}, 0.095$ mmol) by Method B gave $\mathbf{5 f}(E / Z, 20: 80 ; 7.0 \mathrm{mg}, 60 \%)$.

$(E, E ; E, Z)-1-(4-M e t h o x y p h e n y l)-4-p h e n y l-1,3-b u t a d i e n e ~(6 c)$. Treatment of 4c $(30.0$ $\mathrm{mg}, 0.071 \mathrm{mmol})$ with $\beta$-bromostyrene $(14 \mu \mathrm{L}, 20 \mathrm{mg}, 0.11 \mathrm{mmol})$ by Method A gave 6c $(1 E, 3 E / 3 E, 1 Z, 27: 73 ; 11 \mathrm{mg}, 66 \%)$ with data as reported. ${ }^{19 \mathrm{a}, 24} \mathrm{GC}-\mathrm{MS}\left(t_{\mathrm{R}} 21.6 \mathrm{~min} ; Z / E ; t_{\mathrm{R}}\right.$ $23.1 \mathrm{~min} ; E / E) \mathrm{m} / z 236\left(100, \mathrm{M}^{+}\right)$. Anal. Calcd for $\mathrm{C}_{17} \mathrm{H}_{16} \mathrm{O}$ (236.31): C, 86.40; H, 6.82. Found: C, 86.09; H, 6.49.

The isomers of 1,4-diphenyl-1,3-butadiene (3.0 mg, 13\% based on $\beta$-bromostyrene) and the isomers of 1,4-di-(4-methoxyphenyl)-1,3-butadiene $(0.5 \mathrm{mg}, 3 \%)$ were also isolated.

Treatment of $4 \mathbf{c}(30.0 \mathrm{mg}, 0.071 \mathrm{mmol})$ with $\beta$-bromostyrene $(14 \mu \mathrm{L}, 20 \mathrm{mg}, 0.11 \mathrm{mmol})$ by Method B gave $\mathbf{6 c}(1 E, 3 E / 3 E, 1 Z, 18: 82 ; 8.7 \mathrm{mg}, 52 \%)$. The isomers of 1,4-diphenyl-1,3-butadiene ( $2.0 \mathrm{mg}, 9 \%$ based on $\beta$-bromostyrene) and the isomers of 1,4-di-(4-methoxyphenyl)-1,3- butadiene ( $0.4 \mathrm{mg}, 2 \%)$ were also isolated.

$(\boldsymbol{E}, \boldsymbol{E} ; \boldsymbol{E}, \boldsymbol{Z})-1-$ phenyl)-1,3-decadiene (6f). Treatment of $\mathbf{4 f}(25.5 \mathrm{mg}, 0.063 \mathrm{mmol})$ with $\beta$-bromostyrene $(13 \mu \mathrm{L}, 18 \mathrm{mg}, 0.095 \mathrm{mmol} ; E / Z \sim 88: 12)$ by Method A gave $\mathbf{6 f}(1 E, 3 E / 1 E, 3 Z$, $34: 66 ; 9 \mathrm{mg}, 67 \%)$ as a colorless liquid with data as reported: ${ }^{25} \mathrm{GC}-\mathrm{MS}\left(t_{\mathrm{R}} 16.7\right.$ and $\left.17.9 \mathrm{~min}\right)$ $m / z 214\left(22, \mathrm{M}^{+}\right), 129$ (100\%). Anal. Calcd for $\mathrm{C}_{16} \mathrm{H}_{22}$ (214.35): C, 89.65; H, 10.35. Found: C, $89.35 ; \mathrm{H}, 10.02$.

The third isomer of $\mathbf{6 f}(\sim 10 \%)$ was also detected in crude reaction mixture: GC-MS $\left(t_{\mathrm{R}}\right.$ $17.3 \mathrm{~min}) \mathrm{m} / \mathrm{z} 214\left(\mathrm{M}^{+}\right)$.

The isomers of 1,4-diphenyl-1,3-butadiene ${ }^{16,17}(E, E / E, Z, \sim 1: 1 ; 4.0 \mathrm{mg}$ total, $20 \%$ based on $\beta$-bromostyrene) were also isolated: GC-MS ( $t_{\mathrm{R}} 18.7$ and $\left.20.2 \mathrm{~min}\right) \mathrm{m} / z 206\left(100, \mathrm{M}^{+}\right)$. 


\section{References.}

(16) Wang, Z.; Zhang, G.; Guzei, I.; Verkade, J. G. J. Org. Chem. 2001, 66, 3521-3524.

(17) Kang, S.-K.; Kim, J.-S.; Choi, S.-C. J. Org. Chem. 1997, 62, 4208-4209.

(18) Orita, A.; Watanabe, A.; Tsuchiya, H.; Otera, J. Tetrahedron 1999, 55, 2889-2898.

(19) (a) Mitsudo, T.-A.; Fischetti, W.; Heck, R. J. Org. Chem. 1984, 49, 1640-1646, (b) Abu-Youself, I. A.; Hay, A. S. Synth. Commun. 1999, 29, 2915-2922.

(20) Klingelhofer, S.; Schellenberg, C.; Pommerehne, J.; Bassler, H.; Greiner, A.; Heitz, W. Macromol. Chem. Physics 1997, 198, 1511-1530.

(21) (a) Ward, W. J.; McEwen, W. E. J. Org. Chem 1990, 55, 493-500. (b) Takagi, K.; Rousset, C. J.; Negishi, E.-J. J. Am. Chem. Soc. 1991, 113, 1440-1442.

(22) Galamb, V.; Alper, H. Tetrahedron Lett. 1983, 24, 2965-2968.

(23) Hudrlik, P. F.; Bhamidipati, D. M. R. S.; Hudrlik, A. M. J. Org. Chem. 1996, 61, $8655-8658$

(24) van Rossum, A. J. G.; de Bruin, A. H. M.; Nivard, R. J. F. J. Chem. Soc., Perkin Trans. 2. 1975, 1036-1042

(25) (a) Katritzky, A. R.; Denisko, O. V. J. Org. Chem. 2002, 67, 3104-3108. (b) Murakami, M., Ubukata, M.; Ito, Y. Tetrahedron Lett. 1998, 39, 7361-7374. 

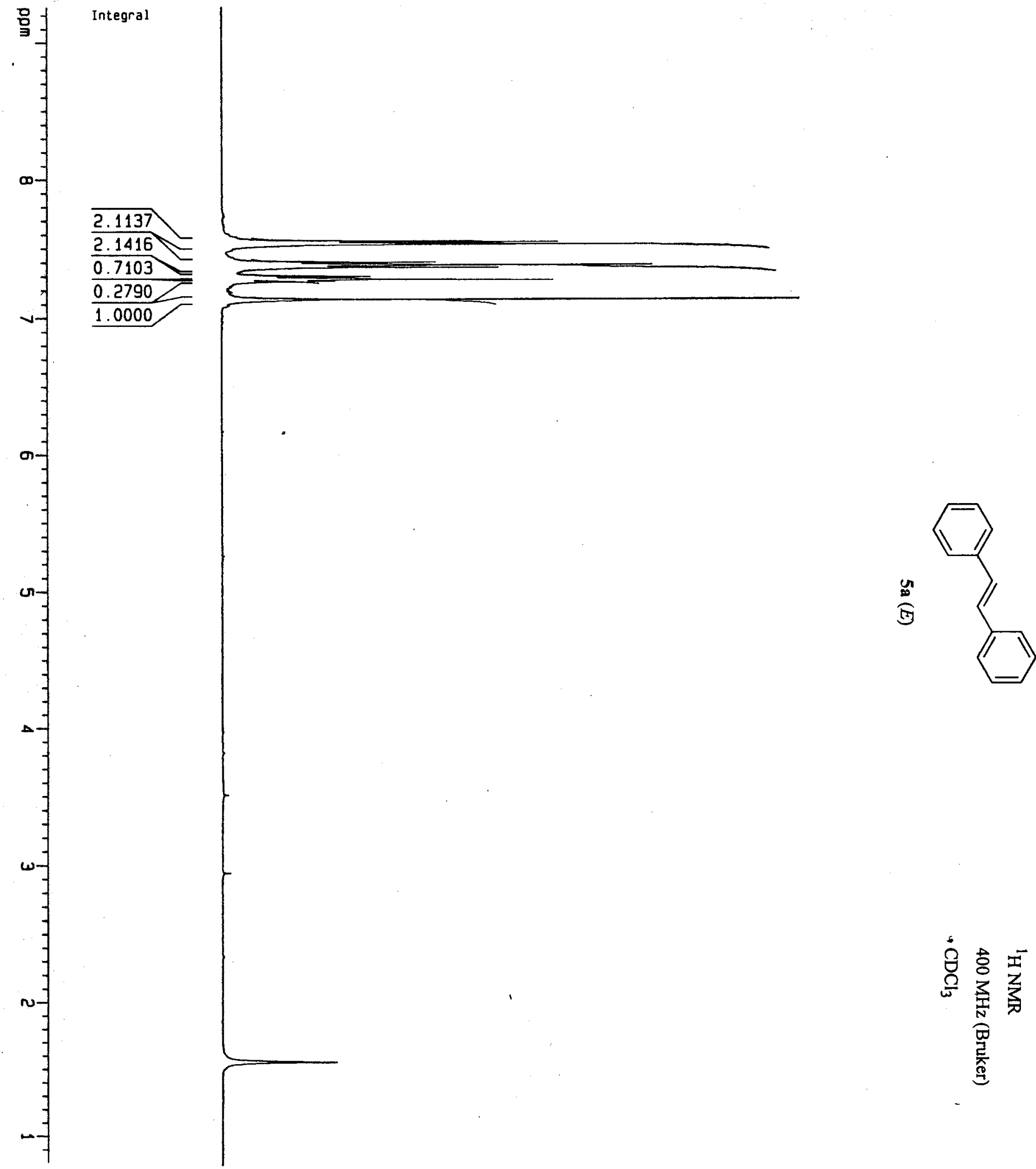

$\stackrel{0}{0}$

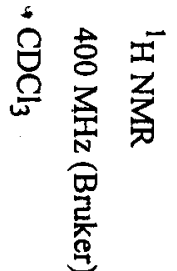




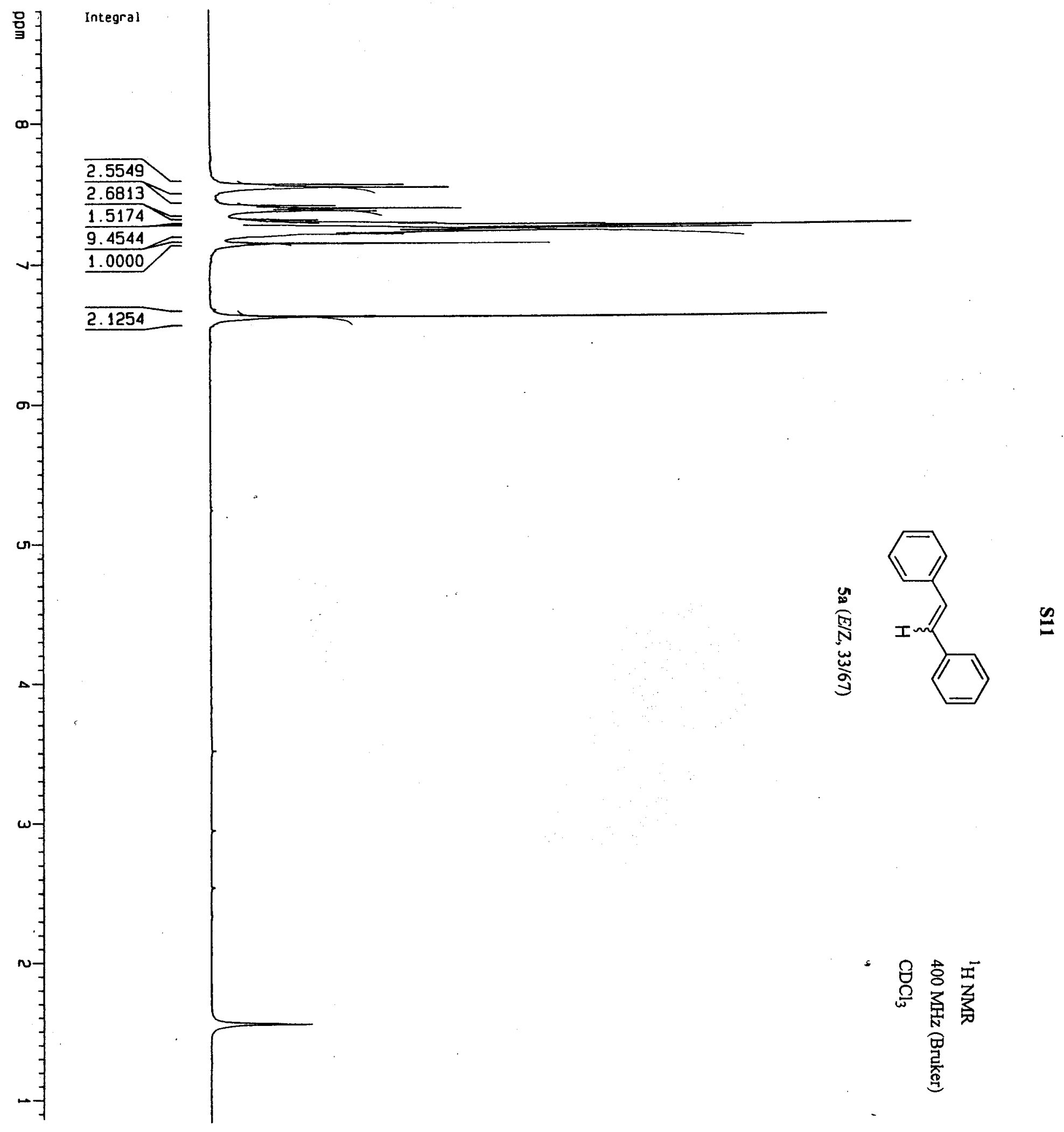




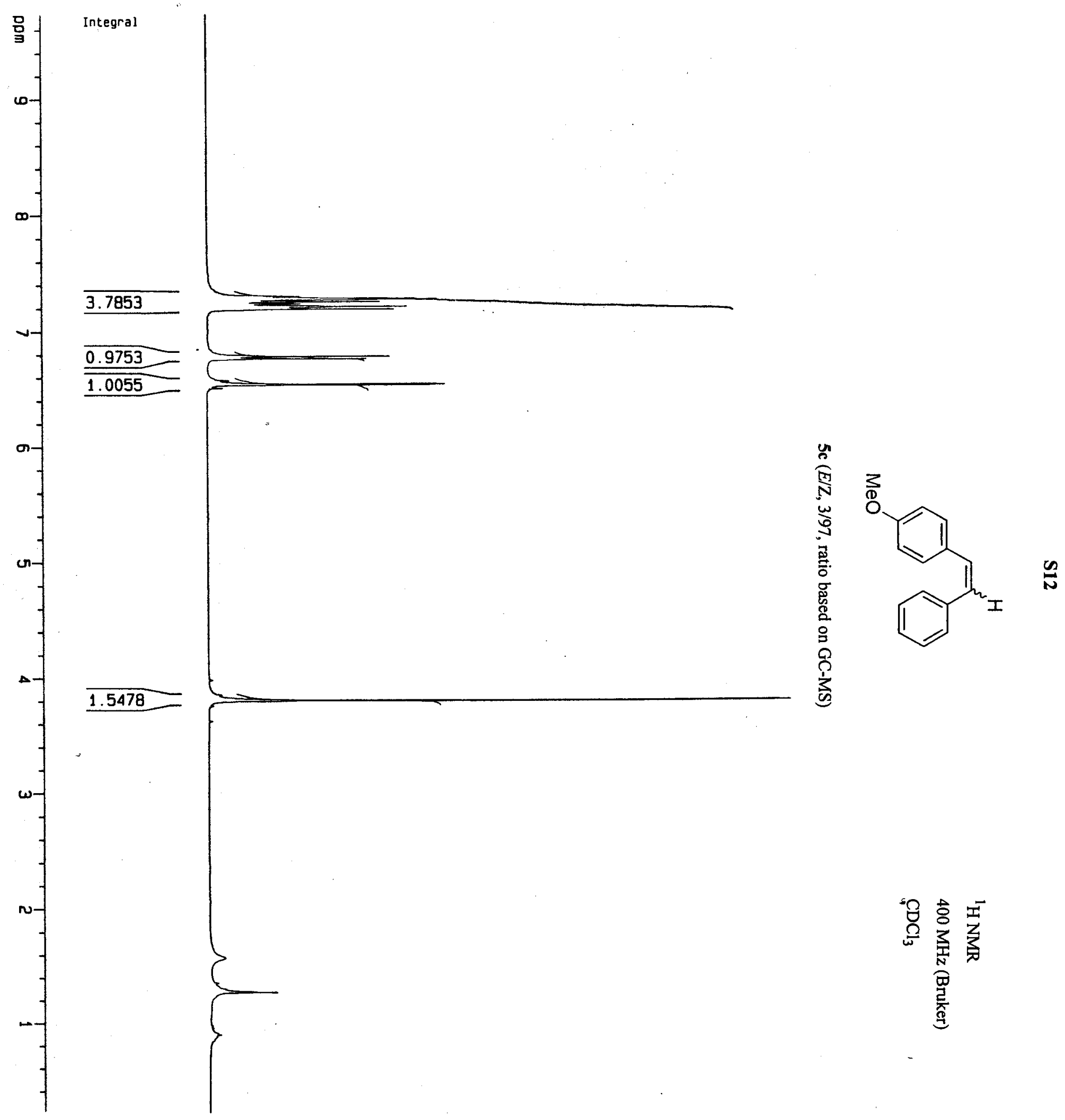




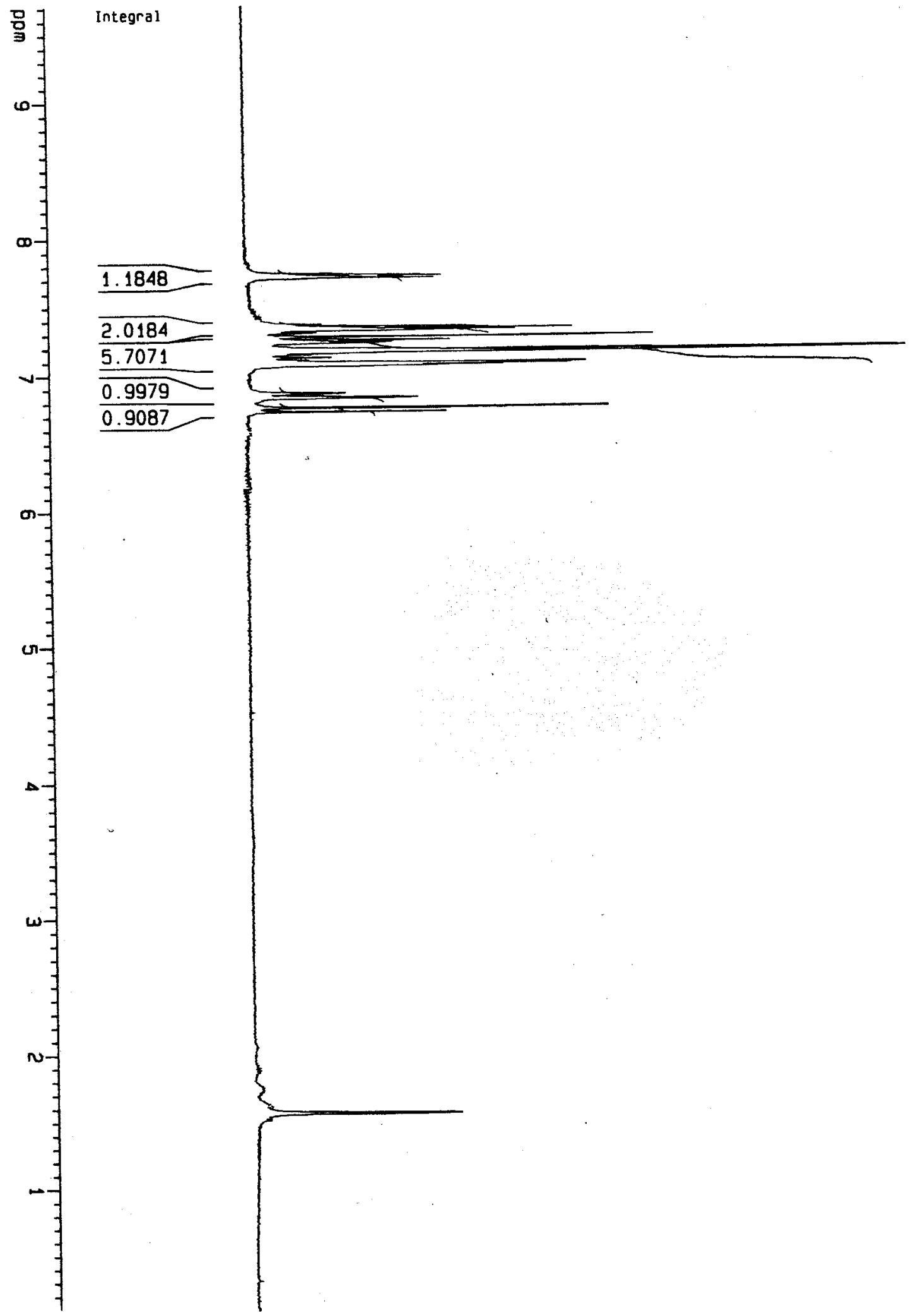

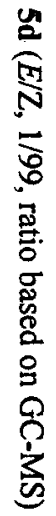




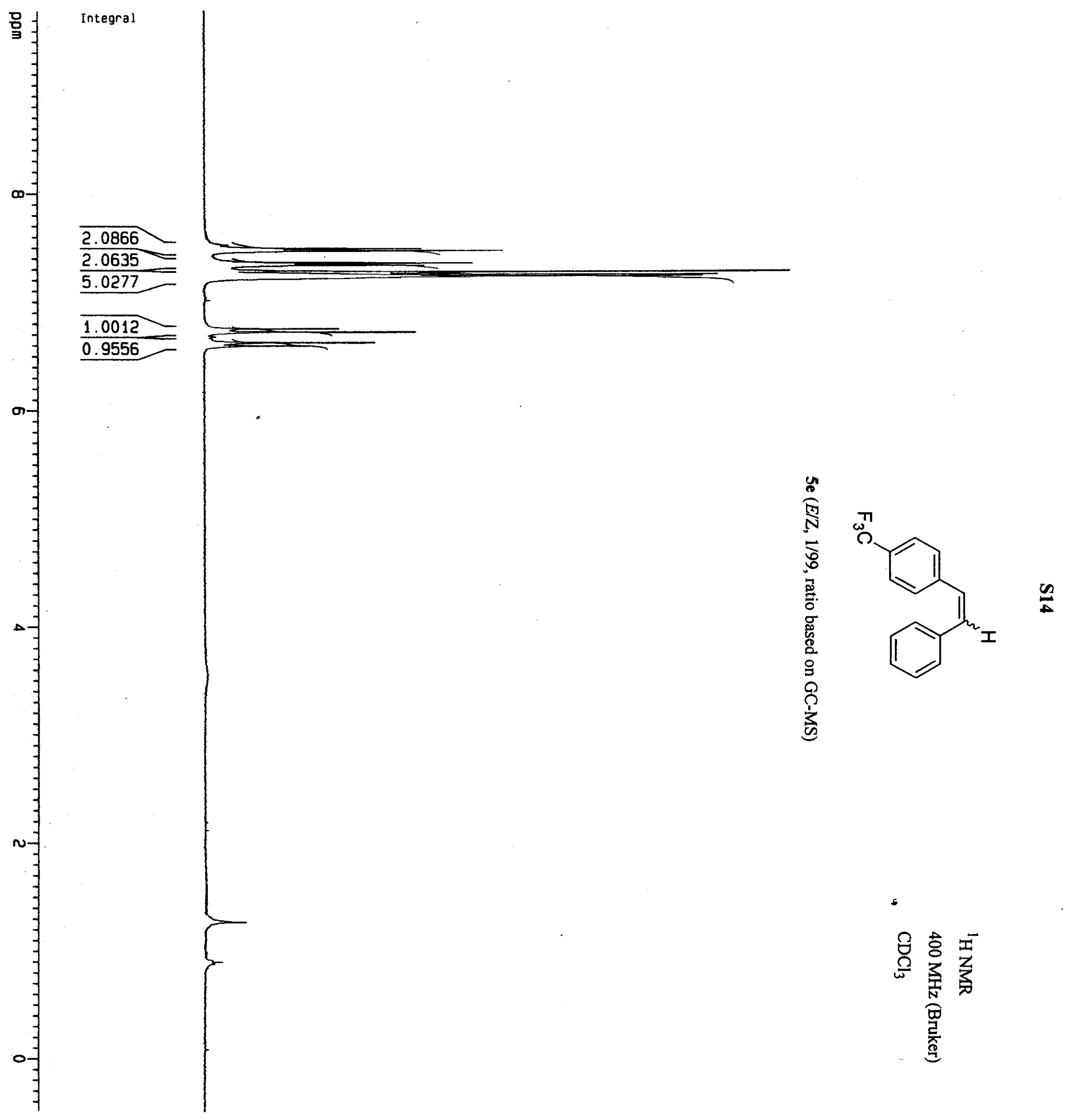

